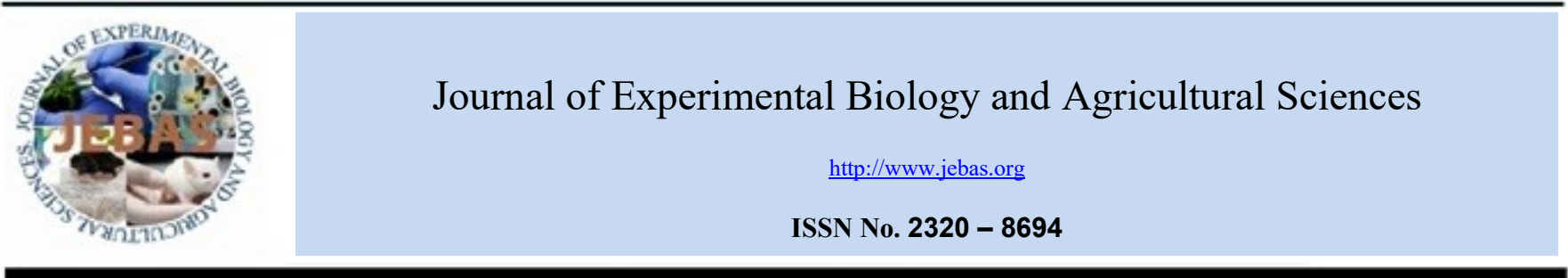

\title{
AUTOPHAGY: AN IMPORTANT PROTEOLYTIC PROCESS FOR THE ENHANCEMENT OF POST-ANTHESIS NITROGEN REMOBILIZATION IN FOOD CROPS
}

\author{
Birendra K Padhan ${ }^{1 *}$, Pandurang R Divte ${ }^{1}$, Nitin Sharma ${ }^{1}$, Rahul Kumar ${ }^{2}$, Somanath Nayak ${ }^{3}$ \\ ${ }^{1}$ Division of Plant Physiology, ICAR-Indian Agricultural Research Institute, New Delhi-110012 \\ ${ }^{2}$ Division of Genetics, ICAR-Indian Agricultural Research Institute, New Delhi-110012 \\ ${ }^{3}$ Division of Agronomy, ICAR-Indian Agricultural Research Institute, New Delhi-110012
}

Received - April 15, 2020; Revision - May 02, 2020; Accepted - June 18, 2020

Available Online - June 25, 2020

DOI: http://dx.doi.org/10.18006/2020.8(3).201.209

\section{KEYWORDS \\ Autophagy \\ Proteolysis \\ Autophagy related proteins (ATG)}

Nitrogen remobilization

\begin{abstract}
Autophagy is an important non-selective cellular degradation process conserved across all kingdom of life. It results in recycling of major biochemical components including proteins that are essential for homeostasis maintenance and physiology. Proteolysis through autophagy yields degradation products such as peptides and amino acids that are remobilized to the developing grains (sink) in major food crops like rice, wheat, maize, etc. Post-anthesis remobilization of nitrogenous compounds to the grains is dependent on pre-anthesis uptake and proteolytic activity during reproductive stage. Proteolysis during grain filling directly affects grain yield and quality. Autophagy is a coordinated proteolytic process that involves upstream signals, autophagy-related protein (ATG) complexes; associated transcription factors (TFs) and various hydrolases that degrade the protein molecules. Therefore, understanding the autophagy mediated proyeolysis and nitrogen remobilization in crops is crucial for enhancing nitrogen use efficiency (NUE) and minimizing $\mathrm{N}$ usage to achieve a sustainable agricultural production system.
\end{abstract}

* Corresponding author

E-mail: kumarbirendra013@gmail.com (Birendra K Padhan)

Peer review under responsibility of Journal of Experimental Biology and Agricultural Sciences.

Production and Hosting by Horizon Publisher India [HPI] (http://www.horizonpublisherindia.in/).

All rights reserved.
All the articles published by Journal of Experimental Biology and Agricultural Sciences are licensed under a Creative Commons Attribution-NonCommercial 4.0 International License Based on a work at www.jebas.org.

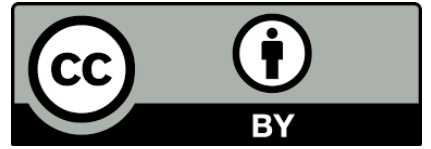




\section{Introduction}

By 2050, doubling in food grain production is required to meet the demand of predicted global population (Fiyaz et al., 2020). But, the available resources for agriculture are diminishing which is a major concern (Godfray et al., 2010). Therefore, increasing resource use efficiency and plant productivity is the only option left behind. To achieve an increase in crop productivity, water and nutrient management are two important strategies. To get a better growth response and higher yield synthetic chemical; especially nitrogenous fertilizers are applied in a larger amount to crops. Nitrogen $(N)$ is a primary nutrient for crop growth and is a constituent of major cellular macro molecules including proteins (Kraiser et al., 2011) Excessive use of $\mathrm{N}$ fertilizers is a threat to the environment and creating pollution. A major challenge of modern agriculture is to reduce the excessive consumption of fertilizers without affecting yield and grain quality. One way to achieve increase in nitrogen economy is to improve plant nitrogen economy through manipulating nitrogen recycling especially nitrogen remobilization, from vegetative plant organs to grains (Zhang et al., 2019; Xuejiao et al., 2020). Grain nitrogen is derived from two different sources; one is pre-anthesis $\mathrm{N}$ uptake and its storage in various vegetative organs, another one source is post-anthesis $\mathrm{N}$ uptake directly by developing grains. First case is predominant because the crop has to complete its life cycle after the reproductive stage and develop grains. Yield and quality of the grain depend not only on the $\mathrm{N}$ absorbed before flowering but also on the mobilization of reused $\mathrm{N}$ from mature leaves during senescence in major food crop like rice, wheat, maize etc (Kichey et al., 2007; Chardon et al., 2012; Li et al., 2015). Major part of the final $\mathrm{N}$ in grains is the result of the remobilization of $\mathrm{N}$ from leaf and other vegetative parts (Chardon et al., 2012). For $\mathrm{N}$ to be remobilized from vegetative tissues to grains, stored proteins in vegetative must be degraded before remobilization. Apart from stem and root, leaf is the major storage organ for proteins. The proteolytic process in leaf tissue increases the source activity for $\mathrm{N}$ remobilization to grains by generating peptides, amino acids (Tegeder \& Masclaux-Daubresse, 2018; Dellero et al., 2020). Various nitrate transporters and amino acid transporters are involved in this mechanism. The conversion of organic nitrogen to inorganic nitrogen through proteolysis and its remobilization to grains is an important determinant for crop yield, productivity and grain quality. Recycling of nutrients through the degradation of sub-cellular components is associated with plant senescence. Autophagy has an important role in plant senescence and nutrient recycling (Doelling et al., 2002), in which cytoplasmic materials and organelles are assembled together and delivered to the vacuole for degradation (Klionsky \& Emr, 2000; Li et al., 2015). Autophagy promotes remobilization of $\mathrm{N}$ to developing grains from vegetative tissues, mostly leaves (Guiboileau et al., 2012), and in autophagy mutants (atg) of Arabidopsis it is found that NUE decreased as compared to wild type under $\mathrm{N}$ deficient conditions (Guiboileau et al., 2013).
From all these observations autophagy mechanism is suspected to be involved in post-anthesi nitrogen remobilization in various crops (Avice \& Etienne, 2014; Masclaux-Daubresse et al., 2017) and widens the scope of autophagy research for scientists. This review highlights the importance of autophagy-mediated proteolytic process in $\mathrm{N}$ recycling and its remobilization to grains, and its role in enhancing nitrogen use efficiency (NUE) of crops.

\section{Protein degradation pathways in plants}

Maintenance of cellular homeostasis is essential for the physiology and survival of plants. It is accomplished by major protein degradation pathways and these pathways are predominant during senescence of plants after reproductive stage. Protein degradation in plants can be broadly classified into; 1) Ubiquitin/proteasome pathway, 2) Chloroplast degradation pathway, 3) Vacuolar and Autophagic (ATG) pathway (Liu et al., 2008; Wang \& Schippers, 2019).

\subsection{Ubiquitin/proteasome pathway}

The ubiquitin/proteasome pathway is important for targeted protein degradation both during normal development and in response to various stresses (Sullivan et al., 2003). This pathway is essential for the elimination of abnormal proteins and the turnover of shortlived proteins. Protein degradation occurs with the help of 76amino acid ubiquitin, which is attached to targeted proteins for degradation and acts as a signal for degradation (Moon et al., 2004). Upon attachment of ubiquitin molecule to the targeted proteins, many such ubiquitin molecules are attached to the proteins to be degraded and lead to the polyubiquitination process. After polyubiquitination, the targeted proteins are degraded with the help of a $26 \mathrm{~S}$ proteasomal complex.

\subsection{Chloroplast degradation pathway}

Chloroplast (CIp) proteins account for $>70 \%$ of all leaf proteins, and it is a major source of $\mathrm{N}$ for mobilization. Chloroplastic proteins (Clp) are degraded either inside the chloroplast itself with help of various proteases or the major proteins inside the chloroplast i.e., Rubisco (accounts for $20-30 \%$ of chloroplast proteins) is packed inside a vesicle along with other chloroplastic protein aggregates called Rubisco Containing Bodies (RCB) and taken to the vacuole for degradation. After degradation, the degraded products are reused in various metabolic processes and also remobilized to developing grains (Guiboileau et al., 2012; Izumi et al., 2017).

\subsection{Vacuolar and autophagic pathway}

Autophagy is an important protein degradation pathway wherein the protein molecules are degraded non-specifically in bulk and maintains cellular homeostasis and survival of cells. Autophagy also results in organelle turn over, degradation of damaged 
proteins, degradation of stored proteins inside the vegetative tissues (vegetative storage proteins), and the degradation of various protein aggregates. Autophagy is found at the basal level in normal plants. During developmental changes like senescence and also during stresses like cellular damage, nutrient deficiency, or pathogen attack autophagic activities are accelerated (Levine \& Klionsky, 2004). Microautophagy and macroautophagy are two important categories of autophagy mechanisms found in plants (Liu et al., 2008). In microautophagy, materials to be degraded are engulfed by the vacuole via the invagination of the tonoplast, followed by pinching off of the membrane to release a vesicle containing the cytoplasmic constituents inside the vacuole lumen. This type of autophagy is found in the case of germinating seeds where the starch granules and storage proteins are degraded inside the vacuoles to provide nourishment to the developing seedlings (Toyooka et al., 2001). Macroautophagy is a common type of autophagy that operates in plants for protein degradation. Therefore, here autophagy refers to macro autophagy. The most extreme form of autophagy is megaautophagy, in which the vacuolar membranes were penetrated or ruptured.

Among all these proteolytic processes discussed, autophagy is thought to play an important role during post-anthesis nutrient recycling especially nitrogen through degradation stored proteins in vegetative tissues and enhances the $\mathrm{N}$ remobilization to developing grains ( $\mathrm{Li}$ et al., 2015).

\section{Autophagy mechanism in plants}

Autophagy is a conserved eukaryotic catabolic process involved in the removal and recycling of damaged or unneeded cellular components in response to various factors. Key regulators such as TOR (target of rapamycin), SnRK1 (sucrose non-fermentingrelated protein kinase-1) and their protein components, targets have been identified in animals, but in plants information regarding transcription factors and regulators is obscure (Liu \& Bassham, 2010; Soto-Burgos \& Bassham, 2017). TORkinase is a key negative regulator of autophagy in plants, whereas the AMPK/SnRK1 kinase acts as a positive regulator. TOR is a serine/threonine kinase and negatively regulates autophagy (DiazTroya et al., 2008) by down regulating autophagic activity upon various stimuli. Before degradation cellular components are sequestered into specialized vesicles called autophagosomes that are delivered to the lytic organelle for degradation, the vacuole in case of plants. Unlike the ubiquitin-proteasome system, autophagy assists in the degradation of large protein complexes and aggregates, as well as non-protein molecules such as nucleic acids, lipid bodies, and sometimes entire organelles. The proteolytic process starts with the formation of a cup-shaped membrane known as phagophore in the cytoplasm that encloses the materials to be degraded i.e., cargo. The cargo is accumulated near the site of degradation with the help of various signaling molecules or adopter molecules. Sequestration of cargo is assisted by a specific type of protein called NBR1 (Near to BRCA1). Recognition and recruitment of specific cargo to autophagosomes are mediated by cargo receptors. The conjugation of the ATG8 proteins with phosphatidylethanolamine (PE) to form the ATG8-PE conjugate that decorates the autophagosome membrane is essential for the expansion of the membrane and for the targeting of the cytoplasm components to be degraded (Noda et al., 2010; MasclauxDaubresse et al., 2017). The phagophore membranes elongate and produce a double-membrane autophagosome. After the formation of double membrane structure it fuses with vacuole (tonoplast membrane). Outer membrane of autophagosome fuses with the tonoplast and the inner membrane containing cargo released into the vacuolar sap as autophagic body for hydrolysis. Inside the vacuole, the autophagic membrane is ruptured first and then the cargo is degraded with the help of vacuolar acid hydrolase, and the degradation products are transferred to the cytosol for reuse.

The process of autophagy operates in a sequential manner starting from the induction of autophagosome membrane formation to the recycling of degradation products as depicted in Figure 1 (Lamb et al., 2013; Ustun et al., 2017; Wang \& Schippers, 2019).

\subsection{Induction}

Induction of autophagy mechanism starts with the upstream signals like senescence or $\mathrm{N}$ starvation or any other environmental stress.

\subsection{Nucleation}

Upon induction by upstream signals the process of nucleation starts with the formation of a membrane called phagophore. The origin of this membrane is obscure, but endoplasmic reticulum (ER) is thought to be the lipid donor for this membrane. Finally this structure leads to the formation of double membrane autophagosome.

\subsection{Vesicle expansion and completion}

Expansion of the phagophore membrane takes place by the lipidation of various autophagy related (ATG) proteins. Among various proteins ATG8 plays an important role in the formation and expansion of the double-membrane structure. Interaction of ATG8-with phosphatidylethanolamine (PE) and the insertion of interacting motif into the membrane of the phagophore involve the ubiquitin pathway (Marshall \& Vierstra, 2018).

\subsection{Autophagosome fusion and degradation}

After completion of autophagosome formation, the complete structure fuses with the vacuole through tonoplast docking and leads to the formation of autophagic body and finally resulted in degradation products. 


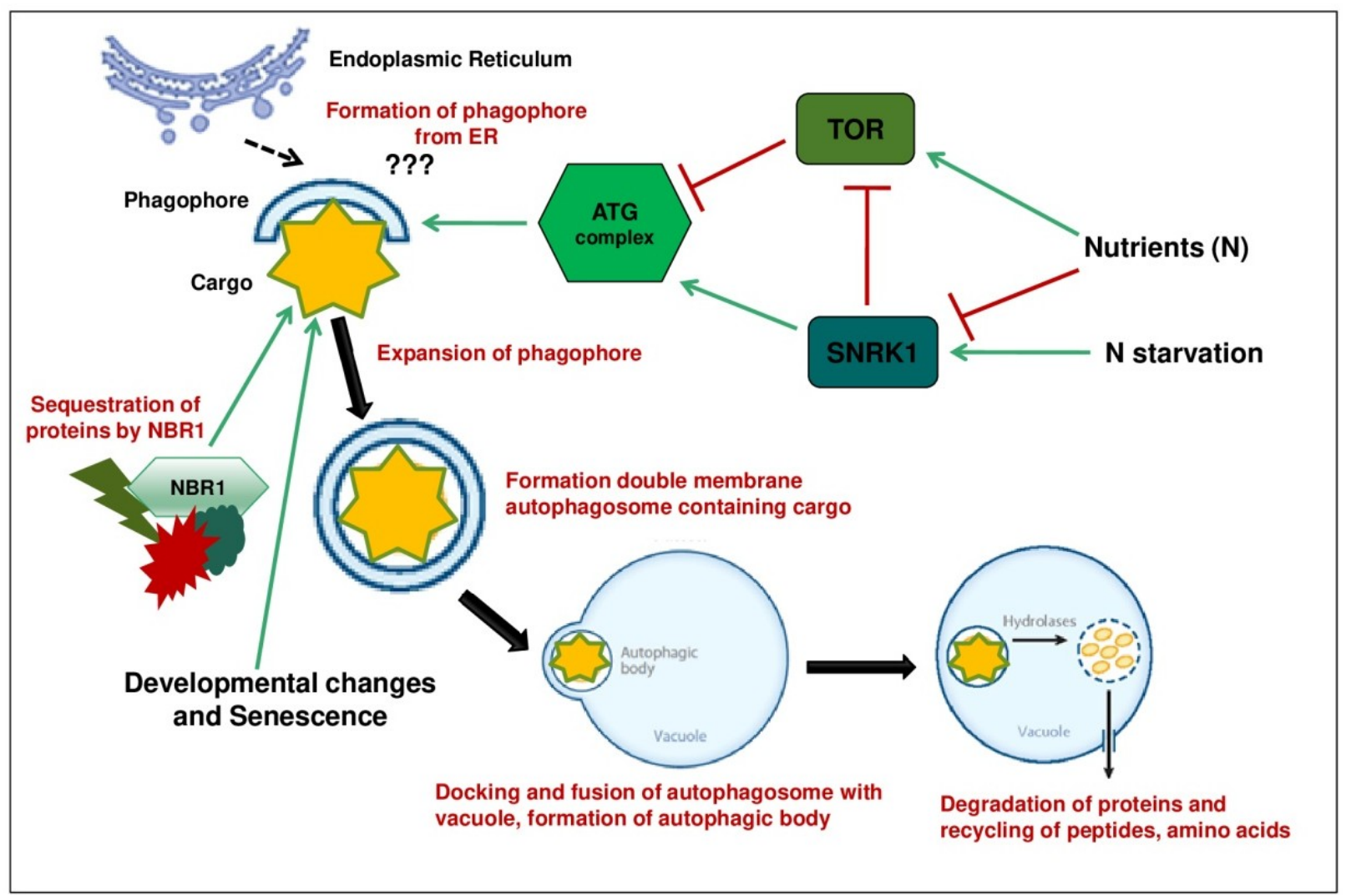

Figure 1 Diagrammatic representation of autophagy machinery induction upon various factors. TOR: target of rapamycin; SNRK1: sucrose nonfermenting-related protein kinase-1; ATG: autophagy related proteins; NBR1: Near to BRCA (breast cancer protein-1)

\subsection{Recycling}

Inside the vacuole, the autophagic membrane is degraded first with the help of lipases and then the cargo is catabolized by different hydrolytic enzymes. Following degradation the degraded products are carried to the cytosol for further use.

\section{Plant autophagy and protein degradation}

Autophagy is found operating at a low constitutive level in all plant cells, but during senescence and stress, the mechanism is upregulated. It differs from other pathways in a way that it aids in non-selective degradation of proteins, nucleic acids, lipids, and starch. Protein degradation through the vacuolar-autophagic pathway is most common in case of plants wherein cellular proteins are recycled. Autophagy machinery is controlled by various upstream signals like TOR kinase (target of rapamycin), Snrk1 (sucrose-nonfermenting related-kinase-1), N starvation, senescence, etc. There are many transcription factors (TFs) associated with autophagy machinery. Formation of autophagosome is assisted with the help of 4 active molecular components, the ATG1 (autophagy-related 1)-ATG13 (autophagy- related 13) kinase complex associated with induction mechanism, the class III phosphatidylinositol 3-kinase (PtdIns3K) complex initiates the nucleation process, and 2 ubiquitin-like conjugation systems for vesicle expansion. ATG8 and ATG12 proteins are involved in phagophore expansion and maturation (Xie et al., 2008; Li et al., 2015).

\subsection{Basal autophagy in plants}

Autophagy activity is found at basal levels in plants during normal growth conditions and maintains homeostasis. . Autophagy eliminates damaged organelles and clear damaged or abnormal proteins that accumulate in the cytoplasm. But in the presence of inhibiter concanamycin A (CA) which inhibits the vacuolar $\mathrm{H}+$ ATPase activity and increases the vacuolar $\mathrm{pH}$ and impaired in hydrolytic activities.

\subsection{Developmental autophagy in plants}

Autophagy is associated with different developmental mechanisms in plants (Janse van Rensburg et al., 2019). During developmental changes like maturity or plant, senescence autophagy helps in 
drainage of stored proteins or other aggregates from senescing leaves to grains through degradative pathways. Autophagy also involves in many developmental processes such as embryonic development, seed germination, shoot and root growth, flower development, and seed generation.

\subsection{Stress-induced autophagy in plants}

Plants have developed various signaling and regulatory mechanisms to cope with environmental changes, including nutrient starvation, salt, drought, and heat stresses (Han et al., 2014; Hilker \& Schmulling, 2019), removing damaged proteins and cellular components (Wang et al., 2018). It acts as both prosurvival and death mechanisms in plants. During programmed cell death (PCD), autophagy activity is accelerated resulting in cellular death to avoid the spread of infection by pathogens, known as the hypersensitive response (HR). During $\mathrm{N}$ starvation autophagy activity is accelerated and results in degradation of reserve materials in vegetative tissues and acts as a pro-survival mechanism.

\subsection{Selective autophagy in plants}

Protein complexes are degraded through receptor mediated selective autophagy process (Yoon \& Chung, 2019) and results in degradation of cellular components, such as various organelles, protein aggregate (Marshall \& Vierstra, 2018). This peocesses are accomplished by the interactions between ATG8 and specific autophagic receptors containing an ATG8- interacting motif (AIM) (Marshall et al., 2015; Farre \& Subramani, 2016; Maqbool et al., 2016). Selective autophagy in plants includes; a. Proteaphagy results in proteasomal complex degradation (Marshall et al., 2015) b. Aggrephagy for the degradation of non-functional protein complexes (Yoon \& Chung, 2019), c. Chlorophagy results in degradation of damaged or redundant chloroplasts (Wada et al., 2009), d. Pexophagy results in degradation of peroxisomes(Huang et al., 2019), e. Reticulophagy for Endoplasmic Reticulum degradation during ER stress, f. Mitophagy for mitochondrial degradation, g. Ribophagy for the degradation of ribosomes when the translation process is at halt.

\section{Autophagy related proteins}

The autophagy process requires the regulated and sequential involvement of a set of autophagy-related (ATGs) proteins that are mostly conserved across all eukaryotic organisms. Autophagy related proteins (ATGs) are conserved across the kingdom but were very first discovered in fungus (yeast) (Tsukada \& Ohsumi, 1993). Till now there are 50 ATGs discovered, 18 are essential for the autophagy core machinery (Yang \& Bassham, 2015). ATG homologs and the complexes are also identified in plants that are regulated by many upstream signals.

\subsection{Functions of ATG8 protein}

ATG8 acts as a central component in autophagy. It is a ubiquitinlike protein conjugated to phosphatidylethanolamine (PE) during vesicle expansion and is located on the autophagosome membrane. ATG8 interacts with multiple autophagy cargo receptors, most importantly through ATG8- interacting motif (AIM) or ubiquitininteracting motif (UIM). Although ATG8 protein is discovered in yeast, there are many orthologs also found in various crop species such as Arabidopsis thaliana, rice (Oryza sativa), maize (Zea mays), tobacco (Nicotiana tabacum), foxtail millet (Setaria italica), and the alga Chlamydomonas reinhardtii (Chung et al., 2009; Perez et al., 2012; Li et al., 2016). In the case of rice 7 ATG8 gene isoforms have been identified so far (OsATGa to OsATGg). $A T G 8$ provides a docking site for autophagic receptors during the fusion of the autophagosome with tonoplast. Overexpression of $A T G 8 s$ from different plants, led to better growth and promoted tolerance to $\mathrm{N}$ limited stress. In Arabidopsis overexpression of different isoforms of ATG8s (AtATG8a, AtATG8e, AtATG8f, AtATG8g) autophagosome number and autophagic flux were increased, stimulated autophagic activity and resulted in enhanced $\mathrm{N}$ remobilization and grain filling (Chen et al., 2019).

\subsection{Functions of NBR1 protein}

Plant analog of this protein is also found during autophagic protein degradation. It is a protein adaptor (Svenning et al., 2011). NBR1 interacts physically with ATG8 and plays an important role in sequestration and aggregation of cargo or proteins for degradation. It also facilitates the enclosure of the autophagosome membrane. Protein sequestration activity of NBR1 can be confirmed by estimating the degradation of proteins through immune blotting. NBR1 synthesis increases when autophagy is stimulated, and the degradation of NBR1 is also stimulated when autophagy flux is increased. During stress conditions or senescence accumulation of NBR1 proteins is increased suggesting its involvement in autophagy and proteolysis (Jung et al., 2020).

\section{Nitrogen remobilization to grains after protein degradation}

Recycling of cellular components is essential for coordination between metabolic pathways and homeostasis. It is also responsible for the removal of debris and replenishes essential nutrients. One of the important mechanisms involved in the recycling of cellular constituents is autophagy which is found to be enhanced to many folds during nutrient starvation. The autophagic recycling process yields amino acids, fatty acids, and sugars, which can be reused later. During senescence of plant organs nitrogen is the important nutrient which is recycled to the grains. This is achieved after the degradation of stored proteins in organs like stem or leaf tissues. $\mathrm{N}$ remobilization in an efficient manner after proteolysis in stored organs thus increases the competitiveness of 
plants under $\mathrm{N}$ deficient conditions. $\mathrm{N}$ import into developing seeds is largely derived from the remobilization of amino acids produced by the breakdown of proteins synthesized before the onset of the reproductive phase (Patrick \& Offler, 2001). It is well established that the main source for nitrogen recycling and remobilization during leaf aging is the chloroplast (Masclaux et al., 2000). Chloroplast protein contributes nearly $70-80 \%$ of total leaf proteins and the enzyme present in chloroplast; Rubisco is a major protein of chloroplast, i.e., 20-30\% of total leaf proteins. Apart from Rubisco other leaf proteins also participate in the proteolytic process, and remobilization of degraded products to developing grains takes place through phloem tissues. ${ }^{15} \mathrm{~N}$ isotope experiment to study the $\mathrm{N}$ remobilization from leaves to grains has shown that the remobilization activity is declined significantly in the mutant of ATG18a RNAi, atg5, and atg9 compared with wild type plants (Guiboileau et al., 2012). Ammonium, amino acids, and proteins accumulated in the rosette leaves of the atg mutants than in wild type (Guiboileau et al., 2013; Chen et al., 2019).

Nitrogen remobilization is closely associated with crop growth and yield. $\mathrm{N}$ fertilization is not sufficient for the high demand during seed filling as post-anthesis uptake of nutrients decline. $\mathrm{N}$ remobilization is needed from leaves to leaves and leaves to seeds to overcome $\mathrm{N}$ limitation. The differences in the amount of remobilized $\mathrm{N}$ originating from uptake before flowering was mainly due to the capacity of the plant to store $\mathrm{N}$ in sink organs until this period (Barbottin et al., 2005). Nitrogen remobilization is triggered by grain filling activity as the developing grains require nutrients. So, the endogenous $\mathrm{N}$ previously-stored in vegetative parts in the form of nitrate, proteins or amino acids must be remobilized to grains to fulfill this demand. $\mathrm{N}$ in leaves is recycled following protein hydrolysis and exported in the form of amino acids to grains (Masclaux et al., 2000) (Figure 2).

\section{Conclusion and future perspectives}

In agriculture, nitrogen remobilization efficiency is interesting as it can reduce the need for $\mathrm{N}$ fertilization thus preventing its over use. $\mathrm{N}$ remobilization and autophagy mechanism are closely associated as the molecules contain must move from source to sink during the senescence of crops. Autophagy is an important proteolytic process associated with remobilization of $\mathrm{N}$ to grains which occur at a basal level even under favourable conditions and is up-regulated in response to various stresses. Autophagy mechanism functions by the involvement of ATG8 which is a core protein of autophagy machinery and also acts as a signalling molecule. Autophagy influences plant metabolism and is required for efficient remobilization of nutrients from leaves to developing seeds and it has a direct role in crop productivity and yield. Autophagy also modulates other agronomic traits like plant architecture by

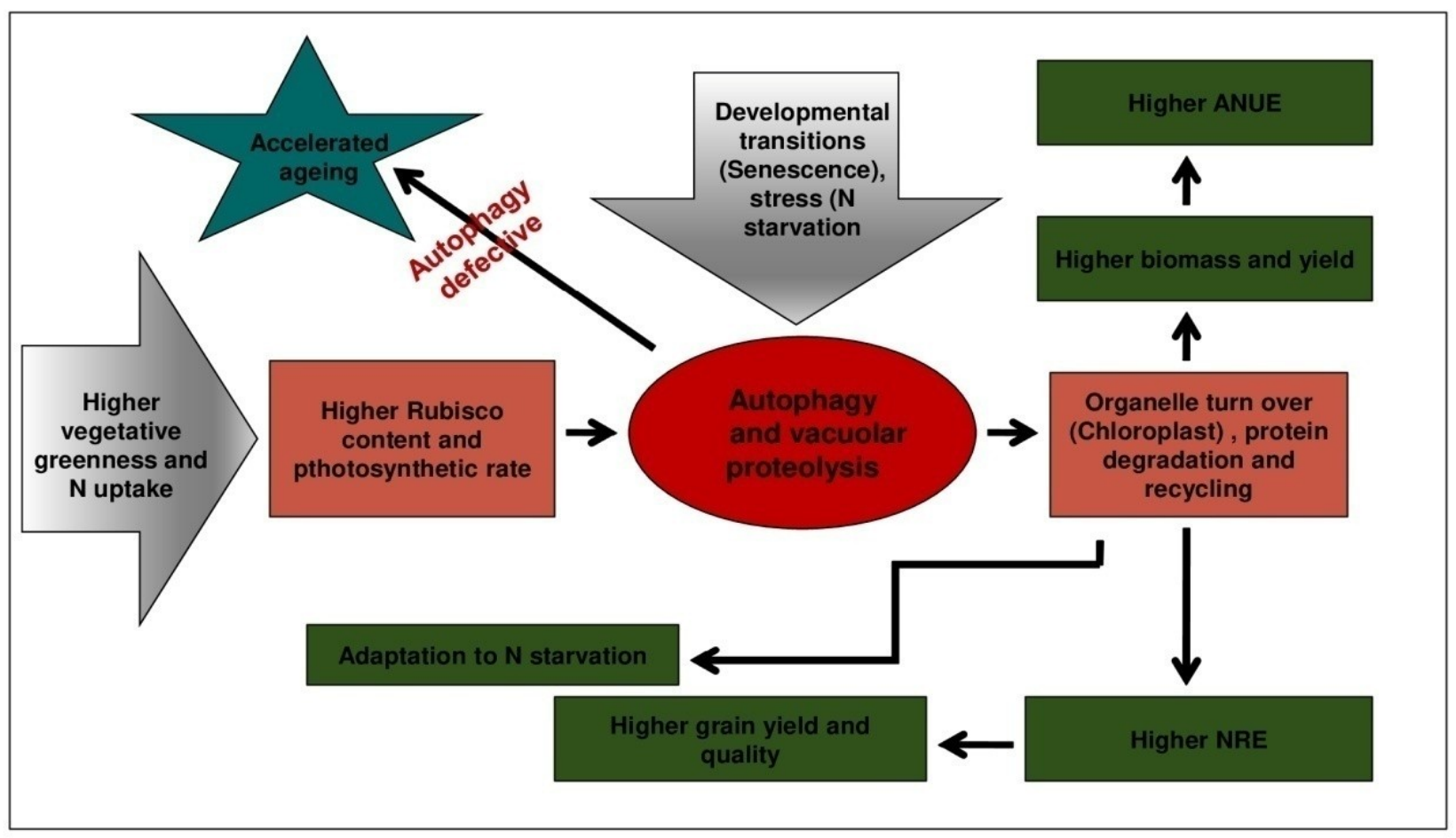

Figure 2 Schematic representation of relationship between crop growth, autophagy and nitrogen remobilization to grains. NRE: nitrogen remobilization efficiency; ANUE: agronomic nitrogen use efficiency 
increasing tiller number, increasing grain number, size, shape, etc. There is a wider scope for autophagic modulators and their targets to enhance autophagic activity and crop yield will be very useful in agriculture. It is evident from various research findings that autophagy is an important mechanism involved in $\mathrm{N}$ recycling through non-selective protein degradation in vegetative tissues and it can be a potential target to enhance $\mathrm{N}$ remobilization efficiency (NRE) and crop yield through plant engineering or marker-assisted breeding to mitigate fertilizer overuse.

\section{Acknowledgement}

All the authors acknowledge ICAR-Indian Agricultural Research Institute, New Delhi for providing support.

\section{Author contributions}

All the authors have contributed irrespective of their area of research and knowledge on the topic.

\section{Conflicts of interest}

All authors declare there is no conflict of interest among them.

\section{References}

Avice JC, Etienne P (2014) Leaf senescence and nitrogen remobilization efficiency in oilseed rape (Brassica napus L.). Journal of Experimental Botany 65: 3813-3824.

Barbottin A, Lecomte C, Bouchard C, Jeuffroy MH (2005) Nitrogen remobilization during grain filling in wheat. Crop Science 45: 1141-1150.

Chardon F, Noel V, Masclaux-Daubresse C (2012) Exploring NUE in crops and in Arabidopsis ideotypes to improve yield and seed quality. Journal of Experimental Botany 63: 3401-3412.

Chung T, Suttangkakul A, Vierstra RD (2009) The ATG autophagic conjugation system in maize: ATG transcripts and abundance of the ATG8-lipid adduct are regulated by development and nutrient availability. Plant Physiology 149: 220-234.

Chen Q, Soulay F, Saudemont B, Elmayan T, Marmagne A, Masclaux-Daubresse C (2019) Overexpression of ATG8 in Arabidopsis stimulates autophagic activity and increases nitrogen remobilization efficiency and grain filling. Plant and Cell Physiology 60: 343-352.

Doelling JH, Walker JM, Friedman EM, Thompson AR, Vierstra RD (2002) The APG8/12-activating enzyme APG7 is required for proper nutrient recycling and senescence in Arabidopsis thaliana. Journal of Biological Chemistry 277 (36): 33105-33114.
Dellero Y, Heuillet M, Marnet N, Bellvert F, Millard P, Bouchereau A (2020) Sink/Source Balance of Leaves Influences Amino Acid Pools and Their Associated Metabolic Fluxes in Winter Oilseed Rape (Brassica napus L.). Metabolites 10 (4): 150. doi: 10.3390/metabo10040150.

Diaz-Troya S, Perez-Perez ME, Florencio FJ, Crespo JL (2008) The role of TOR in autophagy regulation from yeast to plants and mammals. Autophagy 4: 851-865.

Fiyaz RA, Ajay BC, Ramya KT, Kumar JA, Sundaram RM, Rao LS (2020) Speed Breeding: Methods and Applications. Accelerated Plant Breeding, Volume 1 (pp. 31-49). Springer, Cham.

Farre JC, Subramani S (2016) Mechanistic insights into selective autophagy pathways: lessons from yeast. Nature reviews Molecular Cell Biology 17: 537. doi:10.1038/nrm.2016.74.

Godfray HCJ, Beddington JR, Crute IR, Haddad L, Lawrence D, Muir JF, Toulmin C (2010) Food security: the challenge of feeding 9 billion people. Science 327: 812-818.

Guiboileau A, Yoshimoto K, Soulay F, Bataille MP, Avice JC, Masclaux-Daubresse C (2012) Autophagy machinery controls nitrogen remobilization at the whole-plant level under both limiting and ample nitrate conditions in Arabidopsis. New Phytologist 194: 732-740.

Guiboileau A, Avila-Ospina L, Yoshimoto K, Soulay F, Azzopardi M, Marmagne A, Masclaux-Daubresse C (2013) Physiological and metabolic consequences of autophagy deficiency for the management of nitrogen and protein resources in Arabidopsis leaves depending on nitrate availability. New Phytologist 199: 683-694.

Han X, Kumar D, Chen H, Wu S, Kim JY (2014) Transcription factor-mediated cell-to-cell signalling in plants. Journal of Experimental Botany 65: 1737-1749.

Hilker M, Schmulling T (2019) Stress priming, memory, and signalling in plants. Plant Cell \& Environment 42: 753-761. doi: 10.1111/pce.13526.

Huang H, Ullah F, Zhou D X, Yi M, Zhao Y (2019) Mechanisms of ROS regulation of plant development and stress responses. Frontiers in Plant Science 10. doi: 10.3389/fpls.2019.00800.

Izumi M, Ishida H, Nakamura S, Hidema J (2017) Entire photodamaged chloroplasts are transported to the central vacuole by autophagy. The Plant Cell 29: 377-394.

Jung S, Choe S, Woo H, Jeong H, An HK, Moon H, Mun JY (2020) Autophagic death of neural stem cells mediates chronic stress-induced decline of adult hippocampal neurogenesis and cognitive deficits. Autophagy 16: 512-530. 
Janse van Rensburg HC, Van den Ende W, Signorelli S (2019) Autophagy in plants: both a puppet and a puppet master of sugars. Frontiers in Plant Science 10: 14. doi: 10.3389/fpls.2019.00014.

Kraiser T, Gras DE, Gutierrez AG, Gonzalez B, Gutierrez RA (2011) A holistic view of nitrogen acquisition in plants. Journal of Experimental Botany 62: 1455-1466.

Kichey T, Heumez E, Pocholle D, Pageau K, Vanacker H, Dubois F, Hirel, B (2006) Combined agronomic and physiological aspects of nitrogen management in wheat highlight a central role for glutamine synthetase. New Phytologist 169 (2): 265-278.

Klionsky DJ, Emr SD (2000) Autophagy as a regulated pathway of cellular degradation. Science 290 (5497): 1717-1721.

Li F, Lang F, Zhang H, Xu L, Wang Y, Hao E (2016) Role of TFEB mediated autophagy, oxidative stress, inflammation, and cell death in endotoxin induced myocardial toxicity of young and aged mice. Oxidative Medicine and Cellular Longevity. doi: $10.1155 / 2016 / 5380319$.

Levine B, Klionsky DJ (2004) Development by self-digestion: molecular mechanisms and biological functions of autophagy. Developmental Cell 6: 463-477. doi: 10.1016/S15345807(04)00099-1.

Lamb CA, Yoshimori T, Tooze SA (2013) The autophagosome: origins unknown, biogenesis complex. Nature Reviews Molecular Cell Biology 14 (12): 759-774.

Liu J, Wu YH, Yang JJ, Liu YD, Shen FF (2008) Protein degradation and nitrogen remobilization during leaf senescence. Journal of Plant Biology 51 (1): 11-19.

Li F, Chung T, Pennington JG, Federico ML, Kaeppler HF, Kaeppler SM, Vierstra RD (2015) Autophagic recycling plays a central role in maize nitrogen remobilization. The Plant Cell 27 (5): 1389-1408.

Liu Y, Bassham DC (2010) TOR is a negative regulator of autophagy in Arabidopsis thaliana. PLoS One 5 (7). doi: 10.1371/journal.pone. 0011883 .

Masclaux-Daubresse C, Chen Q, Have M (2017) Regulation of nutrient recycling via autophagy. Current Opinion in Plant Biology 39: 8-17. doi: 10.1016/j.pbi.2017.05.001.

Moon J, Parry G, Estelle M (2004) The ubiquitin-proteasome pathway and plant development. The Plant Cell 16 (12): 3181-3195.

Masclaux C, Valadier MH, Brugiere N, Morot-Gaudry JF, Hirel B (2000) Characterization of the sink/source transition in tobacco (Nicotiana tabacum L.) shoots in relation to nitrogen management and leaf senescence. Planta 211: 510-518.
Maqbool A, Hughes RK, Dagdas YF, Tregidgo N, Zess E, Belhaj K, Banfield MJ (2016) Structural basis of host autophagy-related protein 8 (ATG8) binding by the Irish potato famine pathogen effector protein PexRD54. Journal of Biological Chemistry 291 (38): 20270-20282.

Marshall RS, Li F, Gemperline DC, Book AJ, Vierstra RD (2015) Autophagic degradation of the $26 \mathrm{~S}$ proteasome is mediated by the dual ATG8/ubiquitin receptor RPN10 in Arabidopsis. Molecular Cell 58: 1053-1066.

Marshall RS, Vierstra RD (2018) Autophagy: the master of bulk and selective recycling. Annual Review of Plant Biology 69: 173208.

Noda NN, Ohsumi Y, Inagaki F (2010) Atg8-family interacting motif crucial for selective autophagy. FEBS letters 584 1379-1385.

Patrick JW, Offler CE (2001) Compartmentation of transport and transfer events in developing seeds. Journal of Experimental Botany 52: 551-564.

Perez-Perez ME, Lemaire SD, Crespo JL (2012) Reactive oxygen species and autophagy in plants and algae. Plant Physiology 160: 156-164.

Sullivan JA, Shirasu K, Deng XW (2003) The diverse roles of ubiquitin and the $26 \mathrm{~S}$ proteasome in the life of plants. Nature Reviews Genetics 4: 948-958.

Soto-Burgos J, Bassham DC (2017) SnRK1 activates autophagy via the TOR signaling pathway in Arabidopsis thaliana. PLoS One 12 (8). doi: 10.1371/journal.pone.0182591.

Svenning S, Lamark T, Krause K, Johansen T (2011) Plant NBR1 is a selective autophagy substrate and a functional hybrid of the mammalian autophagic adapters NBR1 and p62/SQSTM1. Autophagy 7: 993-1010.

Tegeder M, Masclaux-Daubresse C (2018) Source and sink mechanisms of nitrogen transport and use. New Phytologist 217 (1): 35-53.

Toyooka K, Okamoto T, Minamikawa T (2001) Cotyledon cells of Vigna mungo seedlings use at least two distinct autophagic machineries for degradation of starch granules and cellular components. The Journal of Cell Biology 154: 973-982.

Tsukada M, Ohsumi Y (1993) Isolation and characterization of autophagy-defective mutants of Saccharomyces cerevisiae. FEBS letters 333: 169-174.

Ustun S, Hafren A, Hofius D (2017) Autophagy as a mediator of life and death in plants. Current Opinion in Plant Biology 40: 122-130. 
Wang Y, Mandal AK, Son YO, Pratheeshkumar P, Wise JT, Wang L, Chen Z (2018) Roles of ROS, Nrf2, and autophagy in cadmium-carcinogenesis and its prevention by sulforaphane. Toxicology and Applied Pharmacology 353: 23-30.

Wang H, Schippers JH (2019) The role and regulation of autophagy and the proteasome during aging and senescence in plants. Genes 10 (4): 267. doi: 10.3390/genes 10040267.

Wada S, Ishida H, Izumi M, Yoshimoto K, Ohsumi Y, Mae T (2009) Autophagy plays a role in chloroplast degradation during senescence in individually darkened leaves. Plant Physiology 149 885-893. doi: 10.1104/pp. 108.130013.

Xuejiao Z, Zhenwen Y, Zhang Y, Shi Y (2020) Nitrogen supply modulates nitrogen remobilization and nitrogen use of wheat under supplemental irrigation in the North China Plain. Scientific Reports 10 (1). doi: 10.1038/s41598-020-59877-5.
Xie Z, Nair U, Klionsky DJ (2008) Atg8 controls phagophore expansion during autophagosome formation. Molecular biology of the cell 19 (8): 3290-3298.

Yoon SH, Chung T (2019) Protein and RNA quality control by autophagy in plant cells. Molecules and Cell 42: 285. doi: 10.14348/molcells.2019.0011.

Yang X, Bassham DC (2015) New insight into the mechanism and function of autophagy in plant cells. International Review of Cell and Molecular Biology 320: 1-40. doi: 10.1016/bs. ircmb.2015.07.005.

Zhang M, Fan T, Yang W, Zeng X, Xu X, Xu Y, Xia K (2019) A rice autophagy gene $O s A T G 8 b$ is involved in nitrogen remobilization and grain quality. Research Square doi: $10.21203 / \mathrm{rs} .2 .12993 / \mathrm{v} 1$. 